\title{
Effect of Mechanical Mismatch on the Stress Intensity Factors of Inclined Cracks under Mode I Tension Loading
}

\author{
M.A Azlan ${ }^{1, a}$ and A.E Ismail ${ }^{1, b}$ \\ ${ }^{1}$ Faculty of Mechanical and Manufacturing Engineering, Universiti Tun Hussein Onn Malaysia, \\ Batu Pahat, 86400 Johor, Malaysia \\ aazwir@uthm.edu.my, bemran@uthm.edu.my
}

Keywords: Mechanical Mismatch, Stress Intensity Factors, Inclined Cracks, Mode I Loading.

\begin{abstract}
This paper presents numerical analysis of stress intensity factors (SIFs) of inclined cracks due to mechanical mismatches. According to literature survey, tremendous amounts of SIFs can be found elsewhere. However, the SIFs for inclined cracks are difficult to obtain especially when mechanical mismatch at the crack interface are considered. ANSYS finite element program is used to model the cracks embedded in plain strain plates. The cracks are oriented at the interface between two different materials and subjected to mode I tension loading. It is showed that when mechanical mismatches are introduced the mode I SIFs reduced and on the other hand mode II SIFs increased. When the cracks are inclined, the mode I SIFs diverged but it is not for mode II SIFs and gradually increased when compared with the normal cracks.
\end{abstract}

\section{Introduction}

The stress intensity factor (SIF) is one of the most important cracks driving force in characterizing the structures containing cracks. It is successfully used to ensure the reliability and integrity of mechanical structures. Tremendous amount of works available in discussing the role of cracks in determining the structural strength [1-3]. However, most of these works focused more on the normal cracks [4-8]. According to author's knowledge, the works on the inclined cracks embedded in dissimilar materials are difficult to find [9-11]. Therefore, the main objective of this paper is to analyze the behavior of inclined edge cracks in dissimilar materials subjected to mode I tension loading.

In this paper, three inclined angles of cracks are used $10^{\circ}, 30^{\circ}$ and $40^{\circ}$ including the normal cracks. Different relative crack length, $a / L$ are used in the range of $0.1-0.7$. In order to study the effect of mechanical mismatch on the SIFs, three types of mismatches are used 1.0, 5.0 and 5.0. The numerical model is developed using ANSYS finite element program. Interaction domain integral is used to calculate the SIFs and therefore used to characterize the crack tip. Then the roles of slanted angles, relative crack length and mechanical mismatches on the SIFs are analyzed and discussed.

\section{Methodology}

Two inclined ends of plates are perfectly bonded and it is assumed a single edge crack initiated at one side of the plate as shown in Figure 1(a). The plates are modeled using ANSYS finite element program by considering the plate fulfilled a plain strain condition as in Figure 1(b) with an enlarged area around the crack tip (Figure 1(c)). The plate is constrained in $y$-direction along the lower end of the plate. Additionally, it is also constrained in $x$-direction at a bottom-left lower end point in order to prevent body's rotation. The plate is loaded in tension at the upper end of the plate. In order to study the influence of mechanical mismatches, two types of plates of different material properties are used where the inclination line is as an interface between materials. Iso-parametric 20 nodes singular elements are used around the crack tip while the rest region is meshed with irregular elements. 


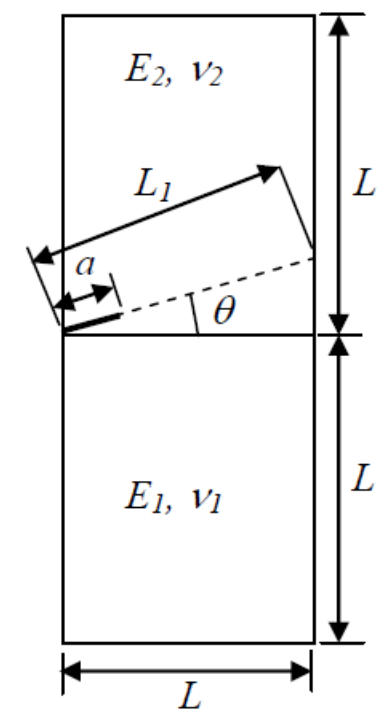

(a)



(b) (c)

Figure 1 (a) Schematic diagram for a slanted crack, (b) Finite element model of the slanted crack and (c) The enlarged area at the crack tip.

In order to calculate the SIFs at the crack tip, J-integral based on the domain integral method is used. Then the calculated J-integral is converted into SIFs using interaction integral formulation. The chosen of energy based method to determine SIFs rather than traditional technique which is displacement extrapolation method due to ease of implementation in numerical model. The values of SIFs are then normalized into dimensionless SIFs or also known as geometrical correction factor, $F$ according to Equations (1) and (2):

$$
\begin{gathered}
F_{I}=\frac{K_{I}}{\sigma \sqrt{\pi a}} \\
F_{I I}=\frac{K_{I I}}{\sigma \sqrt{\pi a}}
\end{gathered}
$$

where, $F$ is geometrical correction factor, $K$ is a stress intensity factor, $\sigma$ is a applied stress and $a$ is a crack depth. Subscript I and II represent type of failure mode I and II. Then $F$ is plotted against relative crack length, $a / L$ and slanted angle, $\theta$.

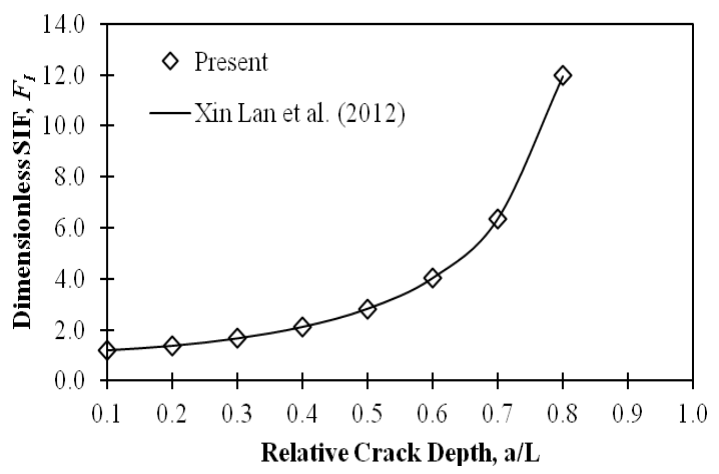

Figure 2 Present model validation with the existing model.

Before any further analysis, the present numerical model shown in Figure 1 is validated with the existing model [4]. However, the validation is performed only for normal crack since there are no 
SIFs for slanted surface crack available in open literature. Figure 3 depicted the SIF comparisons between present and existing crack models. It is showed that both models are well agreed with each others for two crack aspect ratios, $a / b$.

\section{Results and Discussion}

The effect of mechanical mismatches on the mode I SIFs of slanted cracks are presented in Figure 3. For a comparison purpose, Figure 3(a) shows the SIFs for normal cracks of the dissimilar joint plates where the SIF distributions are almost flattened compared with the SIFs obtained from the slanted cracks. It is also revealed that for shallow cracks $(a / L<0.2)$, the SIFs seemed to have a single value. However, when it is reached $(a / L=0.3)$, all the SIFs diverged as the mechanical mismatches increased as shown in Figures 3(b)-3(d). On the other hand, increasing mechanical mismatches decreased the SIFs. While, the SIFs for mechanical mismatch greater than 5, the SIFs are not significantly decreased. The SIF distributions are diverged when higher inclined angles are used.

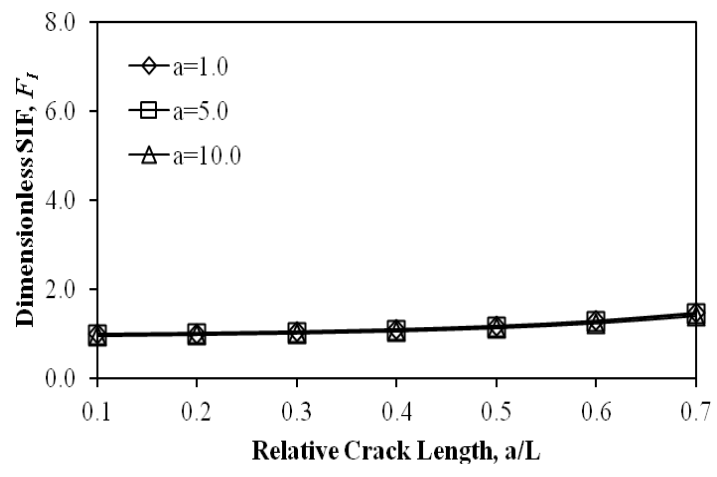

(a)

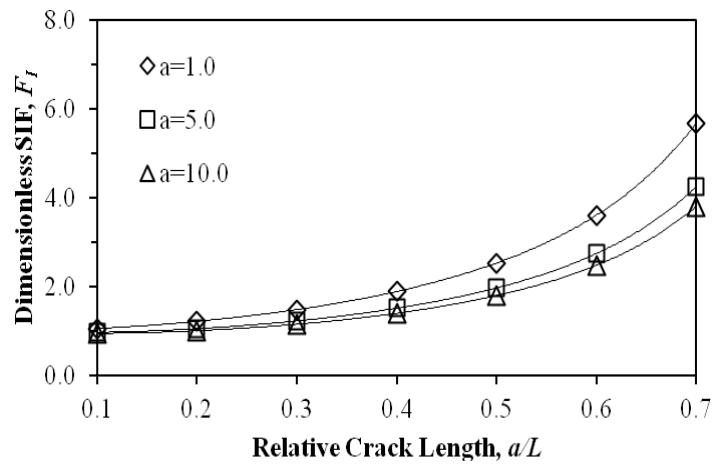

(c)

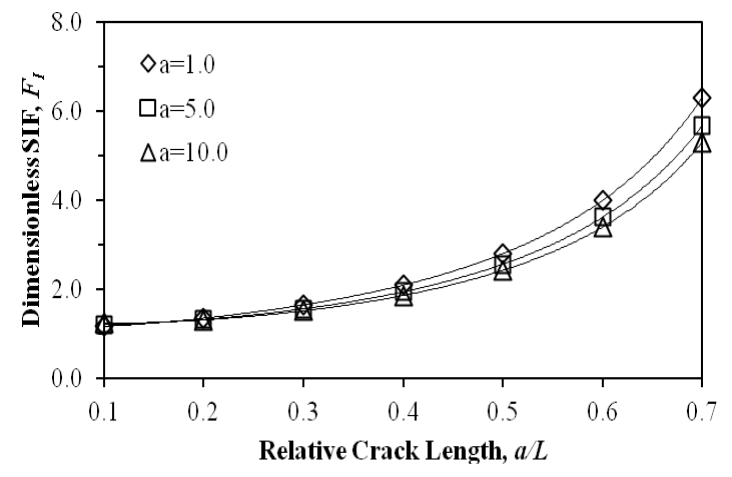

(b)

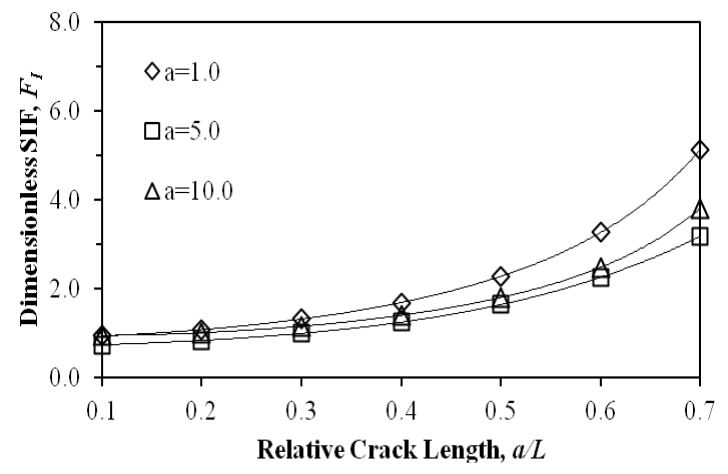

(d)

Figure 3 Effect of mechanical mismatches on the mode I SIFs of different slanted angle, $\theta$, (a) $0^{0}$, (b) $10^{\circ}$, (c) $30^{\circ}$ and (c) $40^{\circ}$.

The influence of mechanical mismatches on the mode II SIFs for inclined cracks are presented in Figure 4. Similar behavior of mode II SIFs compared with mode I SIFs except that all these type of SIFs have an opposite effect. Figure 4(a) reveals the SIFs for interfacial normal cracks in dissimilar materials. It is seemed that the SIFs have no effect when the crack length is increased. The introductions of inclined cracks have induced larger values of mode II SIFs. This is indicated that the slanted cracks capable to reduce the structural performances. According to Fig. 4, both mechanical mismatch and inclined angles played an important role in determining the behavior of SIFs. For a lower value of inclined angles, the behavior of mode II SIFs are obvious. However, when higher angles are used, the SIFs seemed closing to each others. 


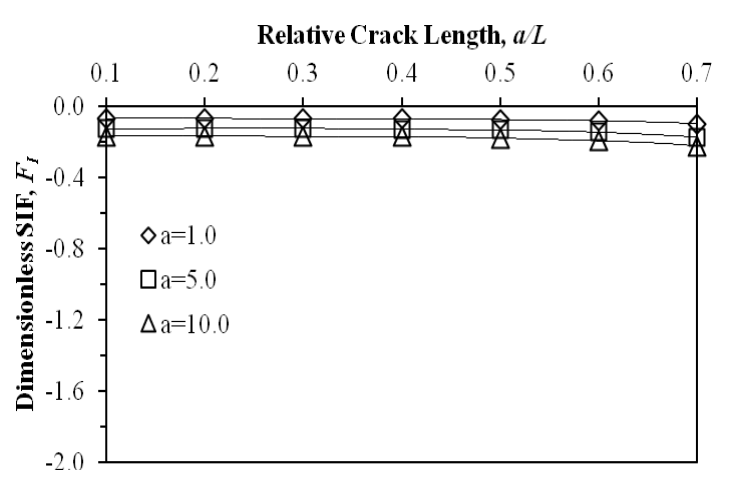

(a)

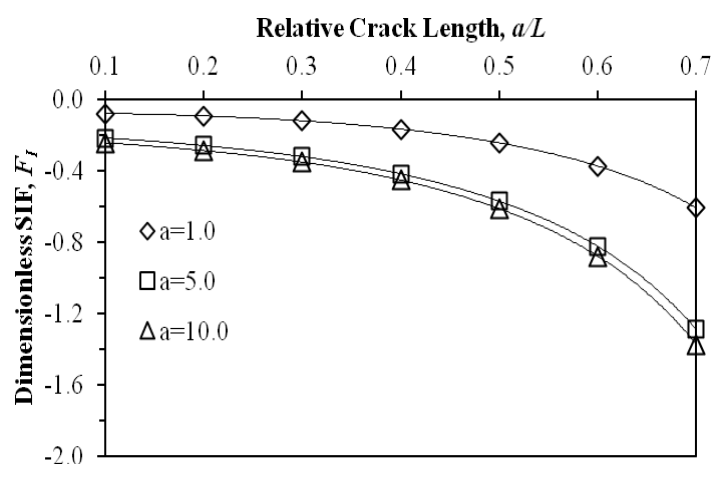

(c)



(b)

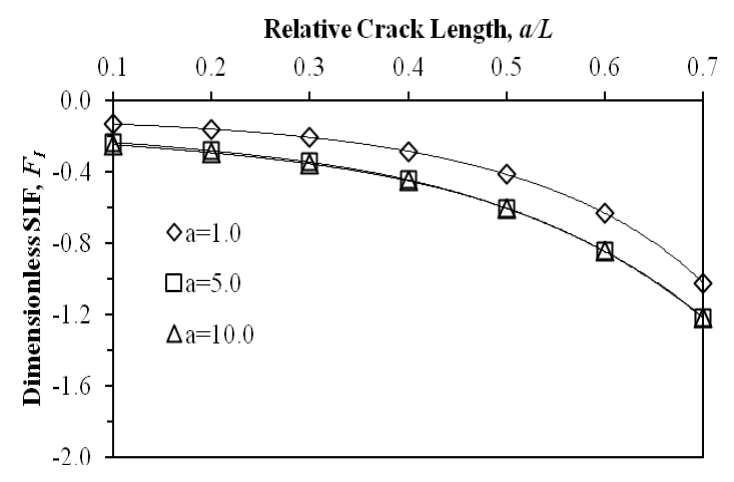

(d)

Figure 3 Effect of mechanical mismatches on the mode II SIFs of different slanted angle, $\theta$, (a) $0^{0}$, (b) $10^{\circ}$, (c) $30^{\circ}$ and (c) $40^{\circ}$.

\section{Summary}

Two types of materials are perfectly bonded and numerically modeled using ANSYS finite element program. Three different mechanical mismatches and inclined angles are used and then the stress intensity factors at the crack tips are evaluated when the crack length is increased. All the results are compared with the normal cracks for identical crack conditions. Therefore, several conclusions can be drawn as follows:

1. The mode I SIFs for normal cracks are almost flattened compared with the inclined cracks. For inclined cracks, the mode I SIFs increased as the crack length increased.

2. Mode II SIFs are also increased but in an opposite direction of mode I SIFs. However, mode II SIFs have no significant effect of mechanical mismatch is greater than 5.0 especially for inclined angle greater than $40^{\circ}$.

\section{Acknowledgement}

Author acknowledged Universiti Tun Hussein Onn Malaysia (UTHM) and Office of Research, Innovation, Commercialization and Consultancy (ORICC) for sponsoring this paper using Vot. 1001. 


\section{References}

[1] A.E Ismail. Stress intensity factors of three parallel edge cracks under bending moments. IOP Conf. Series: Materials Science and Engineering 50 (2013) 012020.

[2] A.E Ismail, A.K Ariffin, S. Abdullah, M.J Ghazali. Stress intensity factors under mode III loadings. International Review of Mechanical Engineering 7(3) (2013) 578-582.

[3] A.E Ismail. Development of J-integral prediction model for surface cracks in rounds bars under combined loadings. Applied Mechanics and Materials 315 (2013) 665-669.

[4] X. Lan, N.A Noda, Y. Zhang and K. Michinaka. Single and double edge interface crack solutions for arbitrary forms of material combination. Acta Mechanica Solida Sinica 25(4) (2012) 404-416.

[5] X. Lan, N.A Noda, K. Mithinaka, Y. Zhang. The effect of material combinations and relative crack size to the stress intensity factors at the crack tip of a bi-material bonded strip. Engineering Fracture Mechanics 78 (2011) 2572-2584.

[6] K. Oda, X. Lan, N.A Noda and K. Michinaka. Effect of arbitrary bi-material combination and bending loading conditions on stress intensity factors of an edge interface crack. Int. J. Structural Integrity 3(4) (2012) 457-475.

[7] T, Matsumto, M. Tanaka, R. Obara. Computational of stress intensity factors of interface cracks based on interaction energy release rates and BEM sensitivity analysis. Engineering Fracture Mechanics 65 (2000) 683-702.

[8] X. Yan, C. Miao. Interaction of multiple cracks in a rectangular plate. Applied Mathematical Modeling 36 (2012) 5727-5740.

[9] Jafar Albinmousa, Nesar Merah, Shafique M.A Khan. A model for calculating geometry factors for a mixed-mode I-II single edge notched specimen. Engineering Fracture Mechanics 78 (2011) 3300-3307.

[10] A.E Ismail, A.K Ariffin, S. Abdullah, M.J Ghazali. Ungkapan kamiran-J retak permukaan pada bar silinder padu kenaan beban ragam I. Jurnal Teknologi (Sciences and Engineering) 68 (1) (2014) 7-17.

[11] A.E Ismail, A.K Ariffin, S. Abdullah, M.J Ghazali. J-integral evaluation of surface cracks in round bar under mode III loadings. Research Journal of Applied Sciences, Engineering and Technology 7 (10) (2014) 1985-1993. 